1. MBBS

House Officer

Department of Internal Medicine

King Edward Medical University,

Lahore.

2. MBBS

House Officer

Department of Internal Medicine

King Edward Medical University,

Lahore.

3. MBBS

Post-graduate Resident PGR-2 Department of Surgical Unit-5

Civil Hospital (DHQ) Faisalabad.

Correspondence Address:

Muhammad Haisum Maqsood

House 35, Lalazar Colony,

Agriculture University Faisalabad.

zaighambajwa@yahoo.com

Article received on:

14/01/2019

Accepted for publication:

07/03/2019

Received after proof reading:

31/07/2019

\section{MISDIAGNOSED CASE OF BICKER STAFF BRAINSTEM ENCEPHALITIS.}

\begin{abstract}
Muhammad Haisum Maqsood', Kinza Rubab², Muhammad Zaigham Maqsood ${ }^{3}$
ABSTRACT: Bickerstaff's brainstem encephalitis (BBE) is a rare neurological disease characterized by ophthalmoplegia, ataxia and altered sensorium. Its etiology is thought to be autoimmune in nature and sometimes certain infections precede illness. It is a spectrum of illnesses with Guillain-Barre Syndrome (GBS) and Miller Fischer Syndrome (MFS). We describe an atypical case of BBE which was initially misdiagnosed as meningo-encephalitis. As such, we report this case for its rarity. Informed consent was received from the patient before undertaking and reporting this study.
\end{abstract}

Key words: Bickerstaff, Brain Stem, Encephalitis, Guillain-Barre Syndrome, Neurological disease

Article Citation: Maqsood MH, Rubab K, Maqsood MZ. Misdiagnosed case of bicker staff brainstem encephalitis. Professional Med J 2019; 26(8):1386-1388.

DOI: 10.29309/TPMJ/2019.26.08.3135

\section{INTRODUCTION}

Bickerstaff's brainstem encephalitis (BBE) is a rare neurological disease characterized by ophthalmoplegia, ataxia and altered sensorium. ${ }^{1}$ Its etiology is thought to be autoimmune in nature and sometimes certain infections precede illness ${ }^{2-4}$ It is a spectrum of illnesses with GuillainBarre Syndrome (GBS) and Miller Fischer Syndrome (MFS). ${ }^{5-6}$ We describe an atypical case of BBE which was initially misdiagnosed as meningo-encephalitis. As such, we report this case for its rarity. Informed consent was received from the patient before undertaking and reporting this study.

\section{CASE REPORT}

An eleven-year-old male child, product of nonconsanguineous marriage, developmentally normal, vaccinated student of $8^{\text {th }}$ grade, younger of all siblings presented in ER on $5^{\text {th }}$ Sep 2018 with history of high grade fever for 1 day and altered state of consciousness for 2 hours. The fever was sudden in onset, not associated with rigors and chills or vomiting; but was associated with 1 episode of fits, generalized tonic-clonic with uprolling of eyes, frothing, urinary and fecal incontinence lasting 4 and a half minutes, followed by altered state of consciousness. There was neither any previous history of fits nor any such family history.

On presentation, patient had a GCS of 10/15 (E2 V2 M4), tone was increased, neck rigidity was positive but Kerning's sign was negative. Reflexes were brisk in lower limbs and normal in upper limbs. Plantar reflexes were upgoing. Abdomen was soft, non-distended, liver $2 \mathrm{~cm}$ below right costal margin, soft, left lobe not palpable. There was bilateral equal air entry in chest and normal vesicular breathing was present. Heart sounds were normal. Pulses were palpable and were of good volume. His pulse was $108 /$ min, respiratory rate was $40 / \mathrm{min}$, blood pressure was $100 / 70$ $\mathrm{mmHg}$, temperature was $98 \mathrm{~F}$ and $\mathrm{SpO} 2$ was $98 \%$.

Initially he was managed as meningoencephalitis. Later on, his LFTs were found to be markedly deranged with ALT $1458 \mathrm{U} / \mathrm{L}$, AST $1602 \mathrm{U} / \mathrm{L}$, Bilirubin $1.8 \mathrm{mg} / \mathrm{dl}$. His PT/aPTT were also deranged. His $\mathrm{CBC}$ showed $\mathrm{Hb} 15 \mathrm{~g} /$ $\mathrm{dl}$, platelets 54000 , TLC $5.4 \times 10^{3}$. Persistent thrombocytopenia remained and the patient had altered aspirate due to which lumber puncture 
was not done. He was treated as hepatic encephalopathy. He started to improve in 6 to 7 days with LFTs returning to normal values; and platelets improved after transfusions. PT/aPTT returned to normal level, GCS improved to $12 / 15$ and there were no signs of raised intracranial pressure. Meanwhile, LP was planned and showed a picture of partially treated viral meningitis. Nerve conduction studies and electromyography were done and showed poly-axonal neuropathy. AntiGq1b antibody seropositivity confirmed BBE and that was an important diagnostic test in reaching the diagnosis. Therefore, he was diagnosed as a case of BBE, a very rare post-infectious neurological disease. Thus IVIGs were planned for treatment. Before reaching the final diagnosis Guillain-Barre syndrome was also listed as an important differential.
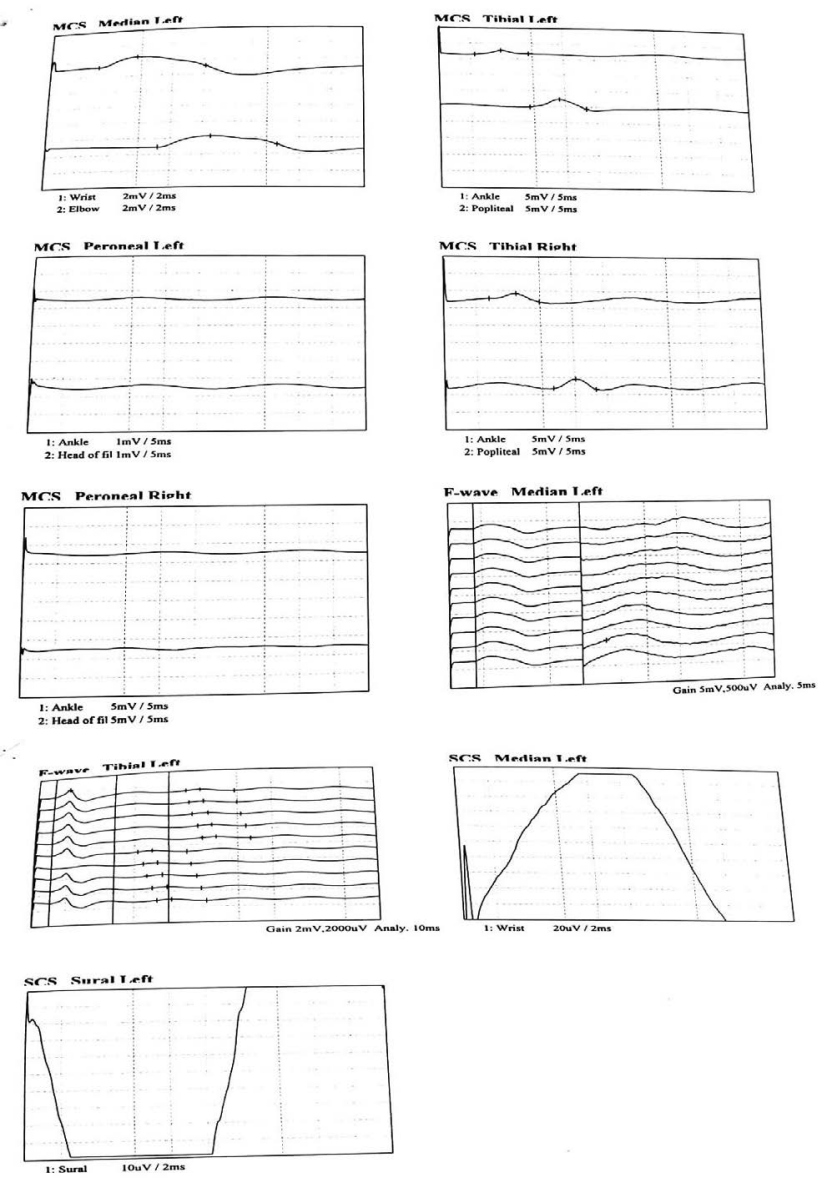

Figure-1\&2. Shows nerve conduction studies. Both peroneal nerves are not excitable. Both tibial nerves show slightly reduced velocities and markedly reduced amplitude of CMAPS. Left median nerve has normal conduction velocity with markedly reduced amplitude of CMAPS. SNAPs are not obtainable in both sural nerves and examined left median nerve. Muscles could not be activated for EMG. In a nutshell, nerve conduction study shows predominantly Axonal Polyneuropathy.

\section{DISCUSSION}

This case of BBE presented without external ophthalmoplegia. Initially, he was misdiagnosed as meningoencephalitis. Anti-Gq1b antibody seropositivity has confirmed a spectrum of BBE and MFS, but MFS has fewer CNS symptoms, as in this case. ${ }^{7}$ So we diagnosed it as BBE. Lack of ophthalmoplegia is thought to be due to rapid progression of altered sensorium. ${ }^{8}$

In this case, presence of anti-GM1 and poly-axonal neuropathy coupled with altered sensorium and positive Babinski sign indicated autoimmune encephalitis compatible with BBE.

Initial presentation of fever should raise concern for infectious origin and it should be addressed accordingly. It is important to differentiate viral encephalitis from BBE, with the symptoms of altered levels of consciousness and headache, as undetermined viral encephalitis may worsen the prognosis. ${ }^{9}$

Due to lack of definitive treatment in BBE, patients treated with IVIG and steroid monotherapies demonstrated shorter times to complete resolution of symptoms from 24 and 60 days, respectively. Other treatments take longer to resolve. ${ }^{10}$

\section{CONCLUSIONS}

The diagnosis of BBE requires history, symptoms, anti-Gq1b antibody seropositivity, MRI and nerve conduction studies because of its atypical presentation.

\section{Copyright@ 07 Mar, 2019.}

\section{REFERENCES}

1. Odaka M, Yuki N, Yamada M, Koga M, Takemi T, Hirata K, Kuwabara S. Bickerstaff's brainstem encephalitis: Clinical features of 62 cases and a subgroup associated with Guillain-Barré syndrome. Brain. 2003 Oct 1 ; 126(10):2279-90.

2. Kanzaki A, Yabuki S, Yuki N. Bickerstaff's brainstem encephalitis associated with cytomegalovirus infection. Journal of neurology, neurosurgery, and psychiatry. $1995 \mathrm{Feb}$; 58(2):260.

3. Hussain A, Flint N, Livsey S, Wong R, Spiers P, Bukhari $S$. Bickerstaff's brainstem encephalitis related to Campylobacter jejuni gastroenteritis. Journal of clinical pathology. 2007 May 18. 
4. Steer AC, Starr M, Kornberg AJ. Bickerstaff brainstem encephalitis associated with Mycoplasma pneumoniae infection. Journal of child neurology. 2006 Jun; 21(6):533-4.

5. Ito M, Kuwabara S, Odaka M, Misawa S, Koga M, Hirata K, Yuki N. Bickerstaff's brainstem encephalitis and Fisher syndrome form a continuous spectrum. Journal of neurology. 2008 May 1; 255(5):674-82.

6. Shahrizaila N, Yuki N. Bickerstaff brainstem encephalitis and Fisher syndrome: Anti-GQ1b antibody syndrome. J Neurol Neurosurg Psychiatry. 2013 May 1; 84(5):576-83.

7. RAJI SHAMEEM MD, SONPAL N, HAMID M, ORSHER S, BHATIA N, WAITZMAN DM, MANDEL S. Bickerstaff's brainstem encephalitis: $A$ rare variant of the antiGq1b antibody syndrome. Practical Neurology. 2013 Sep.
8. Cuneo GL, Grazzini I, Guadagni M, Venturini E, Bianchi A. An atypical Bickerstaff's brainstem encephalitis with involvement of spinal cord. The neuroradiology journal. 2016 Oct; 29(5):396-9.

9. Wakerley BR, Yuki N. A typical bickerstaff brainstem encephalitis: Pitfalls of diagnosis. Pediatric neurology. 2013 Oct $1 ; 49(4)$ :e9.

10. Santoro JD, Lazzareschi DV, Campen CJ, Van Haren KP. Pediatric Bickerstaff brainstem encephalitis: A systematic review of literature and case series. Journal of neurology. 2018 Jan 1; 265(1):141-50.

\begin{tabular}{|c|c|c|c|}
\hline \multicolumn{3}{|c|}{ AUTHORSHIP AND CONTRIBUTION DECLARATION } \\
\hline Sr. \# & Author-s Full Name & \multicolumn{1}{|c|}{ Contribution to the paper } & Author=s Signature \\
\hline 1 & M. Haisum Maqsood & $\begin{array}{l}\text { All contributed in formulation and } \\
\text { writing this case report. }\end{array}$ & H. Huism \\
\hline 2 & Kinza Rubab & \\
\hline 3 & M. Zaigham Maqsood & \\
\hline
\end{tabular}

